\title{
Fabrication of Carbamazepine Cocrystals: Characterization, In Vitro and Comparative In Vivo Evaluation
}

\author{
Muhammad Wasim, ${ }^{1}$ Abdul Mannan, ${ }^{1}$ Muhammad Hassham Hassan Bin Asad $\mathbb{D}^{1,2}$ \\ Muhammad Imran Amirzada, ${ }^{1}$ Muhammad Shafique, ${ }^{3}$ and Izhar Hussain $\mathbb{D}^{1}$ \\ ${ }^{1}$ Department of Pharmacy, COMSATS University Islamabad, Abbottabad Campus Abbottabad 22060, Pakistan \\ ${ }^{2}$ Institute of Fundamental Medicine and Biology, Department of Genetics, Kazan Federal University, Kazan 420008, Russia \\ ${ }^{3}$ Department of Pharmaceutical Science, College of Pharmacy-Boys, Shaqra University, Al-Dawadmi Campus 17441, \\ Shaqra 11911, Saudi Arabia
}

Correspondence should be addressed to Muhammad Hassham Hassan Bin Asad; hasshamasad@yahoo.com and Izhar Hussain; izharhussain@cuiatd.edu.pk

Received 3 November 2020; Revised 25 January 2021; Accepted 5 March 2021; Published 16 March 2021

Academic Editor: Dr. Abdul Ahad

Copyright (C) 2021 Muhammad Wasim et al. This is an open access article distributed under the Creative Commons Attribution License, which permits unrestricted use, distribution, and reproduction in any medium, provided the original work is properly cited.

Carbamazepine (CBZ) is an antiepileptic drug having low bioavailability due to its hydrophobic nature. In the current study, efforts are made to investigate the effect of dicarboxylic acid coformer spacer groups (aliphatic chain length) on physicochemical properties, relative humidity $(\mathrm{RH})$ stability, and oral bioavailability of CBZ cocrystals. Slurry crystallization technique was employed for the preparation of CBZ cocrystals with the following coformers: adipic (AA), glutaric (GA), succinic (SA), and malonic acid (MA). Powder X-ray diffractometry and Fourier-transform infrared spectroscopy confirmed cocrystal preparation. Physicochemical properties, RH stability, and oral bioavailability of cocrystals were investigated. Among the prepared cocrystals, CBZ-GA showed maximum solubility as well as improved dissolution profile (CBZ-GA > CBZ-MA > CBZ-AA > pure CBZ > CBZ-SA) in ethanol. Maximum RH stability was shown by CBZ-AA, CBZ-SA, and CBZ-MA. In vivo studies confirmed boosted oral bioavailability of cocrystals compared to pure CBZ. Furthermore, in vivo studies depicted the oral bioavailability order of cocrystals as CBZ-GA > CBZ-MA > Tegral ${ }^{\circledR}>$ CBZ-AA $>$ CBZ-SA > pure CBZ. Thus, pharmaceutical scientists can effectively employ cocrystallization technique for tuning physicochemical properties of hydrophobic drugs to achieve the desired oral bioavailability. Overall, results reflect no consistent effect of spacer group on physicochemical properties, RH stability, and oral bioavailability of cocrystals.

\section{Introduction}

Pharmaceutical cocrystal (multicomponent system) consists of an active pharmaceutical ingredient (API) and coformer/cocrystal former [1]. The combination of chemically different components in a precise stoichiometric ratio leads to the formation of cocrystal [2]. The noncovalent interactions like $p i-p i$ interactions, Van der Waals forces, and hydrogen bonding are predominantly present between the molecules of crystalline complex $[3,4]$. Pharmaceutical cocrystallization is a good technique for enhancing the physicochemical properties of active pharmaceutical ingredients [5]. Physicochemical properties include solubility, dissolution, melting point, bulk density, hygroscopicity, and compressibility [1-3, 6]. Dissolution and solubility have primary significance owing to their principal role in drug oral absorption [7]. Many cocrystals have been synthesized for modification of physicochemical properties like posaconazole-4-aminobenzoic acid which exhibited high solubility and improved dissolution [8]. Similarly, improved dissolution rate and aqueous solubility were observed by carbamazepine-cinnamic acid cocrystal compared to pure carbamazepine (CBZ) [9]. Cocrystal temozolomide with baicalein enhanced oral drug bioavailability [10]. Carbamazepine-succinic acid cocrystal showed improved physicochemical properties and oral bioavailability [11]. Solubility, dissolution, and stability are the actual significant part of 
the cocrystal studies. Two factors determined the solubility: cocrystal components solvation and crystal lattice energy. Both factors can be influenced to various extents by cocrystallization $[12,13]$. Over the last decade, different studies have been performed on many CBZ (BCS class II) cocrystals to increase its solubility. The low bioavailability of CBZ is due to poor solubility [14]. As stated by biopharmaceutical classification system (BCS), drugs having low solubility despite of high permeability are considered BCS class II drugs. These drugs have dissolution-restricted absorption and low oral bioavailability [15]. Therefore, to get the desired therapeutic effect, CBZ is used generally in a high dose [14].

Many cocrystals have been reported with a series of dicarboxylic acid coformers, for instance, itraconazole cocrystals with coformers like pimelic, adipic, glutaric, succinic, malonic, and oxalic acid; pyrazinecarboxamide with glutaric, succinic, and malonic acid coformers [13]; and CBZ cocrystals with malonic, succinic, glutaric, and adipic acid [16-18]. Different stoichiometry of CBZ cocrystal with coformers malonic $(1: 1 ; 2: 1)$, succinic $(2: 1)$, adipic $(2: 1)$, and glutaric acid $(1: 1)$ has been reported [19]. Pyrazine was cocrystallized with a varying aliphatic chain length (spacer group) of dicarboxylic acid coformers to determine the influence on cocrystal formation and resulting structure of cocrystals [20]. If the spacer group of coformer changes, the conformation as well as the structure of crystal changes. Hence, self-assemblies formation by carboxylic acid coformers having flexible aliphatic spacer group cause changes in alignment and geometries of donors and acceptors [21]. Therefore, it is important to understand the influence of spacer group in dicarboxylic acid coformers $(\mathrm{COOH})-\left(\mathrm{CH}_{2}\right)_{n}-(\mathrm{COOH})$ (Figure 1$)$ : malonic (MA, $n=1$ ), succinic (SA, $\mathrm{n}=2$ ), glutaric $(\mathrm{GA}, n=3)$, and adipic acid (AA, $n=4)$ on physicochemical properties and oral bioavailability of CBZ cocrystals.

\section{Materials and Methods}

2.1. Materials. CBZ was obtained from Tokyo Chemical Industry, Europe. The dicarboxylic acid coformers, MA, SA, and AA, were purchased from Acros Organics, while GA was procured from Sigma-Aldrich. All analytical grade solvents were purchased from commercial sources.

2.2. Cocrystal Preparation. Slurry crystallization method was used for bulk production of each cocrystal in different solvents. Solvents were added in screw-capped glass vials having solid, without reaching full dissolution. The mixtures were stirred for 72 hours at room temperature using a magnetic stirring bar. For further characterization, the resulting powder was rapidly filtered and dried. The detail is given in Table 1.

\subsection{Characterization}

2.3.1. Powder X-Ray Diffractometry. Samples were characterized employing the XRPD method on a diffractometer (Siemens D5000). Samples were irradiated using $\mathrm{Cu}$ as the $\mathrm{X}$-ray source at a current and voltage of $40 \mathrm{~mA}$ and $40 \mathrm{kV}$, respectively. A secondary monochromator was used to allow selection of the $\mathrm{K} \alpha$ radiation of $\mathrm{Cu}(\lambda=1.5418 \AA)$. The samples were measured with a continuous scan rate of $0.01^{\circ}$ s from 2 to $50^{\circ}$ at $2 \theta$.

2.3.2. Fourier-Transform Infrared Spectroscopy. An approximately 3-7 mg of samples was placed on crystal surface (diamond) to obtain the FT-IR spectra by using the PerkinElmer FT-IR spectrophotometer. The FT-IR spectral analysis of finely pulverized samples were carried out at a wavelength range of $450-4000 \mathrm{~cm}^{-1}$.

2.4. In Vitro Studies. In vitro studies like solubility and dissolution were performed for the prepared samples. Concerning dissolution experiments, the initial step was to grind CBZ cocrystals in mortar and pestle to obtain uniform particle size range. In the next step, the instrument (EasyMax 102 Advanced Synthesis Workstation) devised by Mettler Toledo provided with a temperature monitor and a stirrer rotating at $150 \mathrm{rpm}$ to prepare supersaturated CBZ solution by pouring large quantity of sample powder in a flask $(100 \mathrm{~mL})$ having a medium (ethanol) of $40 \mathrm{~mL}$ at $25^{\circ} \mathrm{C}$. Online "React IR iC $10^{\prime}$ " manufactured by Mettler Toledo AutoChem having an ATR crystal (AgX DiComp Fiber Conduit probe) with a size $6.5 \mathrm{~mm}$ attached to the crystallization reactor was used for recording of CBZ IR signature in solution every 15 seconds (50 scans) from $2800 \mathrm{~cm}^{-1}$ to $650 \mathrm{~cm}^{-1}$ till equilibrium. Finally, the React IR data was monitored using the "IR iC 10" software. The samples for solubility measurement were collected when the cocrystal solution reached the saturated state during dissolution experiment. Samples were analyzed by high-performance liquid chromatography (HPLC).

2.5. Relative Humidity Stability Study. Different relative humidity $(\mathrm{RH})$ conditions like $43 \%, 75 \%$, and $98 \%$ were obtained using salt solutions (saturated) of $\mathrm{KCO}_{3}, \mathrm{NaCl}$, and $\mathrm{K}_{2} \mathrm{SO}_{4}$, respectively. The samples were kept in three different $\mathrm{RH}$ conditions for a period of 1 month. The samples were then immediately analyzed for absorption/adsorption of water by TGA-SDTA 851e devised by the Mettler Toledo thermogravimetric analysis (TGA) technique. The TGA thermograms were recorded at a temperature range of $30-150^{\circ} \mathrm{C}$ with a scanning rate of $10^{\circ} \mathrm{C} / \mathrm{min}$ under a nitrogen purge of $50 \mathrm{~mL} \mathrm{~min}^{-1}$. The solid samples of masses around $8-10 \mathrm{mg}$ were analyzed using aluminum oxide crucible. The STARe thermal analysis software was used for data evaluation.

2.6. Filling of Capsule Shells. Though capsule filling is a technical process, for research purposes, filling of capsules does not require specific machines due to small batch size. Therefore, all the suitable size capsules for in vivo pharmacokinetic studies were filled manually.

\subsection{In Vivo Pharmacokinetic Studies}

2.7.1. Animals and Dosing. The protocols used for in vivo pharmacokinetic studies with the approval of "Research Ethical Committee" Department of Pharmacy, COMSATS University Islamabad (CUI), Abbottabad Campus (ref. no PHM-0088/E.C/M5). Healthy rabbits with a body weight 2 $\pm 0.3 \mathrm{~kg}$ were housed and restrained from food about 12 hours prior dosing while being allowed free access to water. 


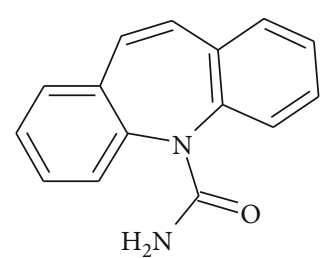

(a)<smiles>O=C(O)[18O]C(=O)O</smiles>

(b)

Figure 1: Chemical structures of (a) carbamazepine and (b) coformers (R-spacer group, $n=1,2 \cdots)$.

TABle 1: Complete detail about cocrystals regarding their preparation.

\begin{tabular}{lcccc}
\hline API & Coformer & Ratio & Solvent & Stirring time (h) \\
\hline CBZ & AA & $2: 1$ & Methanol & 72 \\
CBZ & GA & $1: 1$ & Acetone & 72 \\
CBZ & SA & $2: 1$ & Ethanol & 72 \\
CBZ & MA & $2: 1$ & Methanol & 72
\end{tabular}

Abbreviations: CBZ: carbamazepine, MA: malonic acid, SA: succinic acid, GA: glutaric acid, AA: adipic acid.

All rabbits were randomly divided into five groups, each having six rabbits. Cocrystals and commercial product (pulverized) were filled into capsules and orally administered with $2 \mathrm{~mL}$ of water. Blood samples $(0.5 \mathrm{~mL})$ at different time intervals $(0$ to $18 \mathrm{~h}$ ) were collected in heparinized tubes followed by separation of plasma by centrifugation for $10 \mathrm{~min}$ at $12000 \mathrm{rpm}$ and then stored until further analysis at $-20^{\circ} \mathrm{C}$.

2.7.2. Quantification of CBZ Plasma Concentration. CBZ was quantified in plasma samples as described previously [22] with slight modification, using the HPLC (series 200, PerkinElmer USA) technique. Mobile phase of acetonitrile, methanol, and phosphoric acid buffer $(\mathrm{pH} 6.5) 0.1 \mathrm{~mol} / \mathrm{L}$ at a ratio of 10:30:60 was used. The flow rate and retention time were $1.2 \mathrm{~mL} / \mathrm{min}$ and $3.2 \mathrm{~min}$, respectively. A $250 \times$ $4.6 \mathrm{~mm}$ Supelco ${ }^{\circledR} \mathrm{C}_{18}(5 \mu \mathrm{m}$ particle size $)$ was used as an analytical column. Acetonitrile was added to samples and centrifuged to precipitate proteins. A $20 \mu \mathrm{L}$ supernatant was injected. CBZ concentration was measured at $\lambda_{\max }$ $285 \mathrm{~nm}$ by UV detector.

2.7.3. Data Analysis. Pharmacokinetic (PK) parameters like peak plasma concentration $\left(C_{\max }\right)$ and time to reach peak plasma concentration $\left(T_{\max }\right)$ were measured for noncompartmental model. Trapezoidal rule was employed for the calculation of area under curve $\left(\mathrm{AUC}_{0 \rightarrow t}\right)$ from the concentration-time curve. Equation (1) was used for the calculation of total area under the curve $\left(\mathrm{AUC}_{0 \rightarrow 18}\right)$ :

$$
\mathrm{AUC} 0 \rightarrow 18=\mathrm{AUC} 0 \rightarrow 18+\frac{\mathrm{Ct}}{\mathrm{Ke}},
$$

where $K_{\mathrm{e}}$ is CBZ elimination rate constant (apparent), $C_{\mathrm{t}}$ is CBZ concentration at 18th hour, and ANOVA (one-way analysis of variance) and $t$-test $(p<0.05)$ were used for comparison of PK parameters and statistical analysis of data.

\section{Results}

The powder XRPD patterns of cocrystals are different from its individual components, i.e., $\mathrm{CBZ}$ and respective coformers. The XRPD patterns of CBZ-AA, CBZ-GA, CBZ-SA, and CBZ-MA are perfectly matched with their XRPD powder patterns provided in the Cambridge Structural Database (CSD) as MOXVEB, MOXVOL, XOBCIB, and XOBCEX, respectively (Figure 2). FT-IR spectra of cocrystals are different from its individual components, i.e., pure $\mathrm{CBZ}$ and respective coformers as shown in Figure 3. In vitro studies, like solubility and dissolution behavior of pure CBZ and its four cocrystals, were investigated. The dissolution of cocrystals was improved except CBZ-SA compared to pure CBZ as shown in Figure 4. Cocrystals showed better dissolution whose solubility was enhanced by cocrystallization. The result is summarized in Table 2. The results of RH stability studies of pure CBZ show a weight loss of $10.7 \%$ and $1.9 \%$ at $98 \%$ and $75 \%$ RH conditions, respectively. CBZ-AA and CBZ-SA showed maximum stability with no weight loss. CBZ-GA, the most unstable, even showed a weight loss at $43 \% \mathrm{RH}$ as shown in Figure 5 and Table 3. A probe into the in vivo pharmacokinetic study was conducted in rabbits. Plasma drug profile of pure CBZ, cocrystals, and marketed product is shown in Figure 6. Significant increase in oral bioavailability was observed with cocrystals compare to pure CBZ. CBZ-GA exhibited the highest peak plasma concentration $(7 \pm 0.35 \mu \mathrm{g} / \mathrm{mL})$ with high oral bioavailability $(64 \pm 2.2 \mu \mathrm{gh} / \mathrm{mL})$. Table 4 enlists pharmacokinetic parameters.

\section{Discussion}

The characteristic XRPD peaks of polymorph CBZ-III ${ }^{\#}$ (Figure S1), used in the study that appeared at $2 \theta=15.8$ and 18.6, were found to be in good agreement with reported data [23]. CBZ was cocrystallized with dicarboxylic acid coformers, i.e., AA, GA, SA, and MA to investigate the spacer group effects on physicochemical properties and oral bioavailability of cocrystals. The formation of cocrystals was confirmed by XRPD and FT-IR. The XRPD pattern of samples was in good agreement with their corresponding XRPD patterns in CSD. Figure 2 shows overlays of XRPD pattern of cocrystals consistent with the published data [24]. There are two reported polymorphs of CBZ-MA cocrystals with CSD codes XOBCEX $(2: 1)$ and MOXVUR $(1: 1)$ as shown in Figure S1 [19]; a third form has also been studied, but a single crystal was not yet obtained via solvent crystallization method [25]. CBZ-MA and CBZ-SA have similar packing of CBZ and hydrogen-bonding motif. So these two are isostructural [19]. 

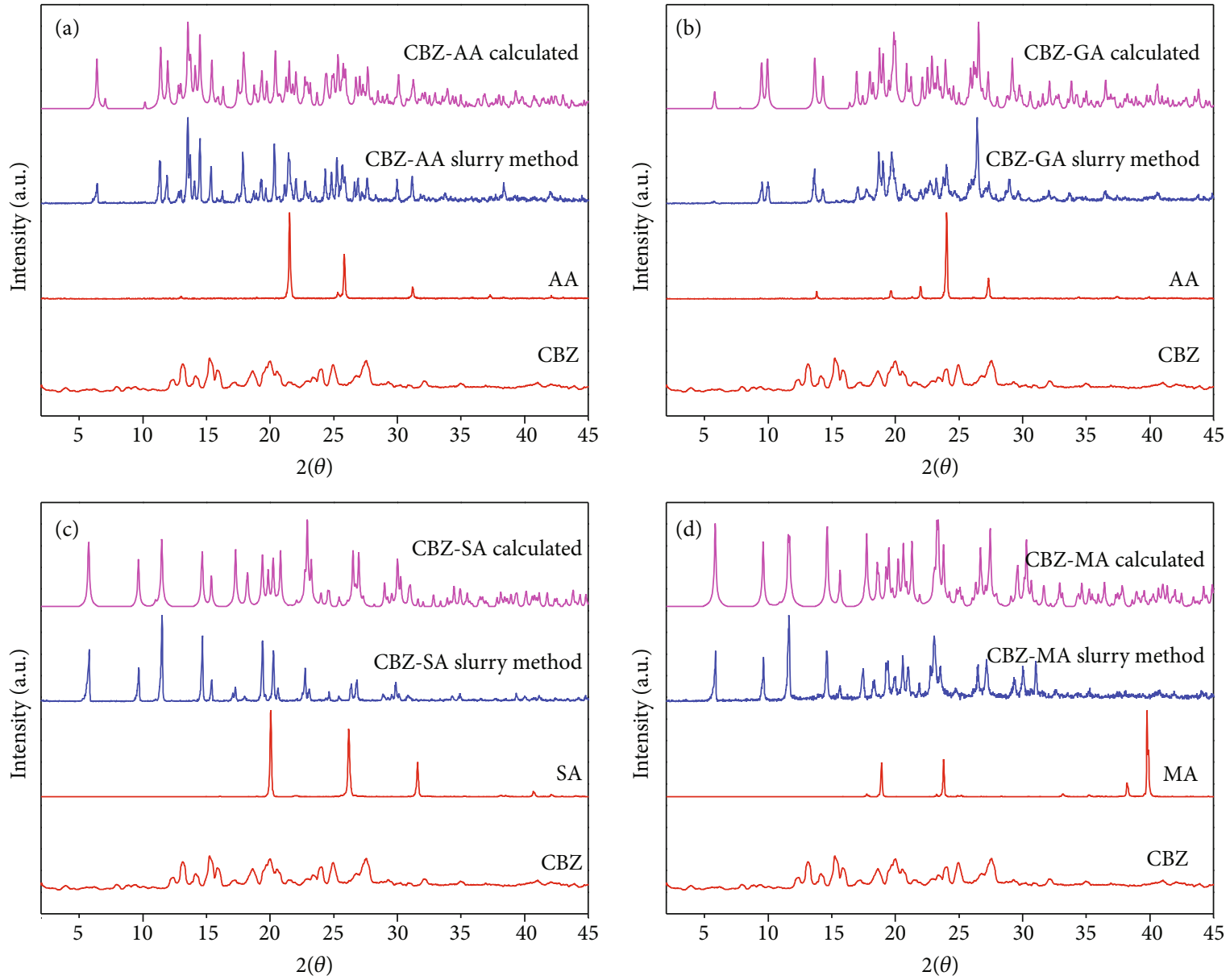

Figure 2: XRPD patterns of (a) CBZ-AA, (b) CBZ-GA, (c) CBZ-SA, and (d) CBZ-MA cocrystals.

Further validation of cocrystals was carried out by FT-IR spectral analysis. The FT-IR spectra of cocrystals are different from their respective components (Figure 3 and Table 5). The pure $\mathrm{CBZ}$ shows the absorption bands of $\mathrm{N}-\mathrm{H}$ stretching and $\mathrm{C}=\mathrm{O}$ stretching at 3465 and $1675 \mathrm{~cm}^{-1}$, respectively [26]. Clear shifts were observed in CBZ-AA, CBZ-GA, CBZ-SA, and CBZ-MA cocrystals due to hydrogen-bond formation. Thus, the XRPD and FT-IR analyses authenticate the formation of CBZ cocrystals with AA, GA, SA, and MA coformers.

In vitro results of $\mathrm{CBZ}$ cocrystals showed that about $60 \%$ of pure CBZ was dissolved in $45 \mathrm{~min}$. But CBZ-SA cocrystal dissolved $52 \%$ in $45 \mathrm{~min}$ being the lowest soluble, whereas dissolution of CBZ-GA, CBZ-MA, and CBZ-AA was 97\%, $90 \%$, and $70 \%$, respectively (Figure 4). CBZ-GA (1:1) showed highest solubility $(24.92 \pm 0.03 \mathrm{mg} / \mathrm{mL})$, while CBZ-SA $(2: 1)$ cocrystal exhibited low solubility of $8.88 \pm$ $0.01 \mathrm{mg} / \mathrm{mL}$ as shown in Table 2. Thus, the CBZ to coformer stoichiometry seems to have no effect on solubility as well as dissolution. The higher final concentration of $\mathrm{CBZ}$ was achieved by all cocrystals except CBZ-SA compared to pure CBZ. This might be due to CBZ-SA cocrystal being low soluble in ethanol [27]. The interesting thing is that maximum solubility of CBZ was observed in 40 minutes by all cocrys- tals, whereas approximately 100-minute longer time was needed for pure CBZ. In cocrystal, the more soluble component (coformer) is usually drawn out of the crystal lattice into dissolution medium [28]. The solubility of dicarboxylic acids series in different solvents including ethanol was determined by Zhang and coworkers to check the effect of "even-odd" number of carbons. The solubility of coformer having odd number of carbons was higher compared to coformer of even number of carbons [29]. Thus, the "even-odd" number of carbons of coformers may also affect the solubility of cocrystal. In our study results, it has been noted that cocrystals having coformer (odd number of carbons) showed higher solubility and vice versa (Table 2). Likewise, in the previous study, the solubility order of coformers (GA > MA > AA > CBZ-SA) in ethanol has been reported [29]. Our results of cocrystal solubility are consistent with the order of solubility of coformers. From a pharmaceutical point of view, a significant dissolution profile was observed by CBZ-GA being the most soluble released about $97 \%$ CBZ in dissolution medium. The enhanced solubility and dissolution of CBZ-GA in the presence of excess coformer is also reported [30]. Past studies also explored that dissolution and solubility can be improved by cocrystallization technique $[8,31-33]$. Likewise, an improved dissolution rate by carbamazepine-cinnamic acid 

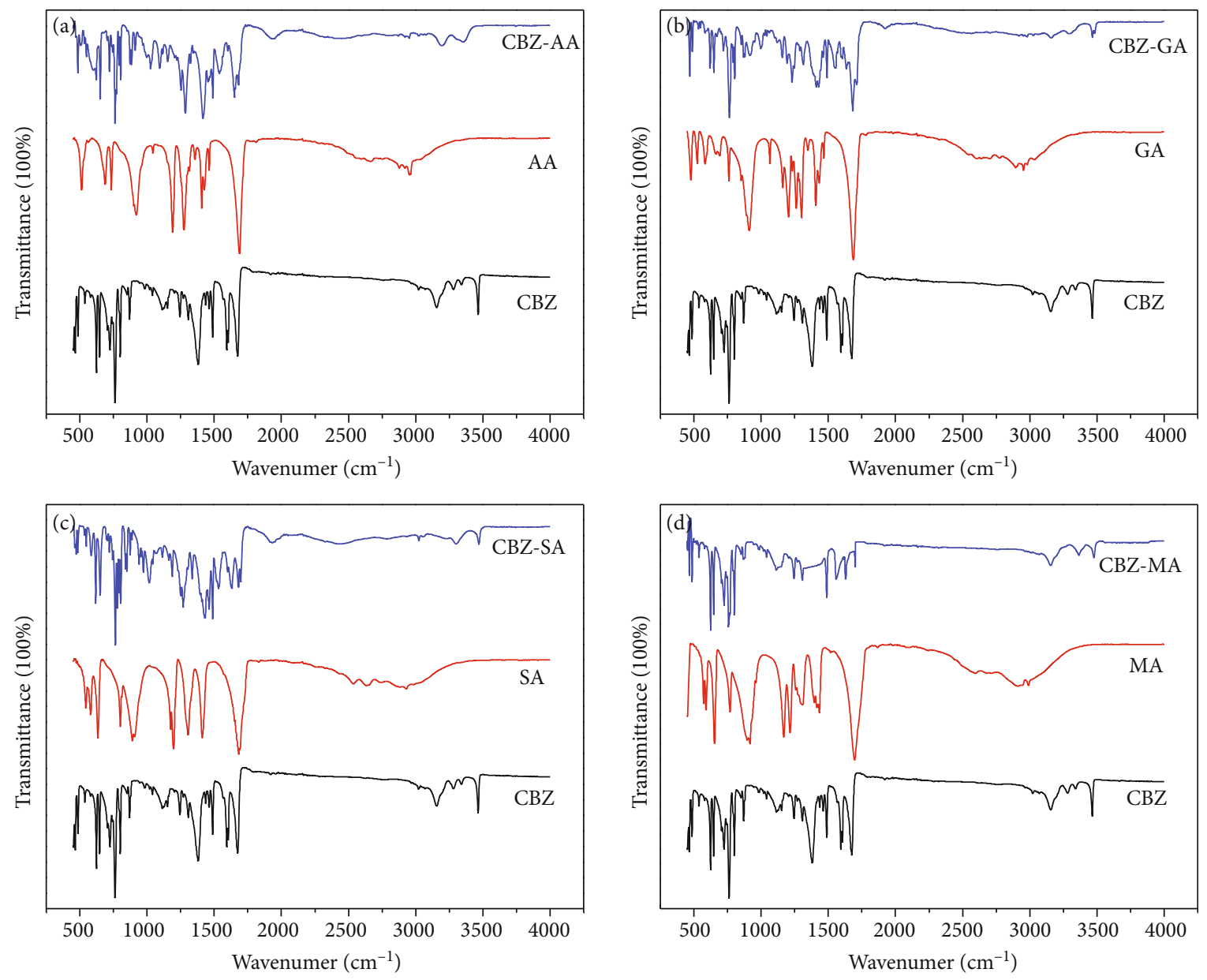

FIgure 3: FT-IR spectra of (a) CBZ-AA, (b) CBZ-GA, (c) CBZ-SA, and (d) CBZ-MA cocrystals.

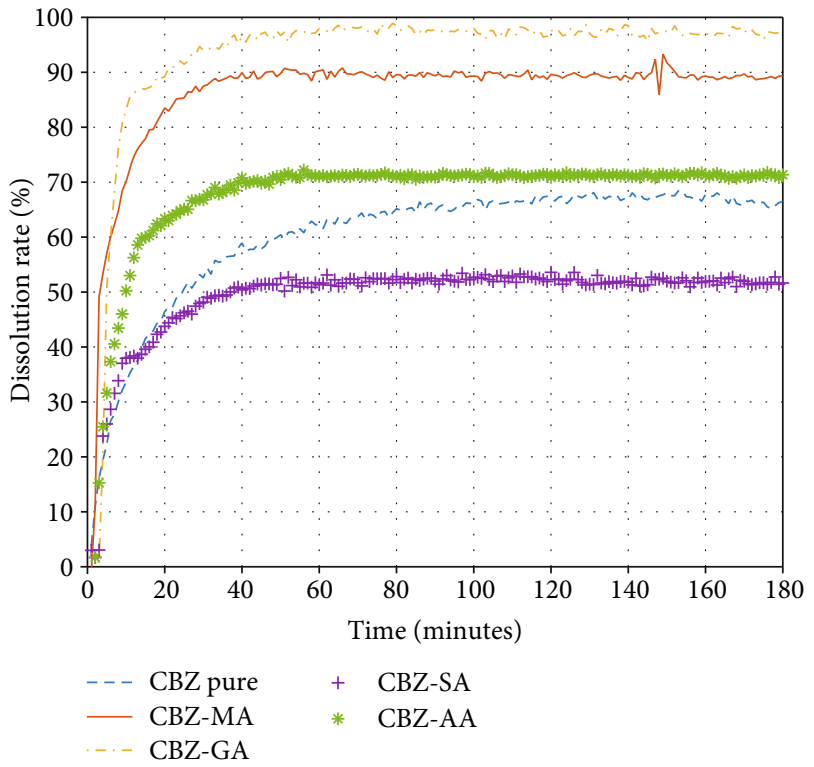

FIGURE 4: Dissolution release profile of CBZ-AA, CBZ-GA, CBZ$\mathrm{SA}, \mathrm{CBZ}-\mathrm{MA}$, and pure CBZ in ethanol.
TABLE 2: Solubility and \% dissolution rate about different cocrystals in ethanol.

\begin{tabular}{lcccc}
\hline S. No. & Samples & $\begin{array}{c}\text { Cocrystal } \\
\text { stoichiometry }\end{array}$ & $\begin{array}{c}\text { Solubility } \\
(\mathrm{mg} / \mathrm{mL})\end{array}$ & $\begin{array}{c}\text { \% dissolution rate } \\
(45 \mathrm{~min})\end{array}$ \\
\hline 01 & CBZ & - & $18.19 \pm 0.06$ & 60 \\
02 & CBZ-MA & $2: 1$ & $24.61 \pm 0.08$ & 90 \\
03 & CBZ-SA & $2: 1$ & $8.88 \pm 0.01$ & 52 \\
04 & CBZ-GA & $1: 1$ & $24.92 \pm 0.03$ & 97 \\
05 & CBZ-AA & $2: 1$ & $22.62 \pm 0.10$ & 70 \\
\hline
\end{tabular}

cocrystal was observed in distilled water compared to pure CBZ [9]. Therefore, enhanced dissolution of cocrystals can be attributed to solubility improvement in the dissolution medium.

Concerning RH stability studies, CBZ-GA showed \% weight loss of $0.7,2.1$, and 6 at 43,75 , and 98\% RH conditions, respectively, whereas CBZ-SA and CBZ-AA showed zero \% weight loss at provided $\mathrm{RH}$ conditions. The weight loss of $11.7 \%$ was observed in CBZ-MA at $98 \% \mathrm{RH}$ and remained stable at 43 and $75 \% \mathrm{RH}$. However, the trend of $\%$ weight loss in pure CBZ was 1.9 and 10.7 at $75 \%$ and 

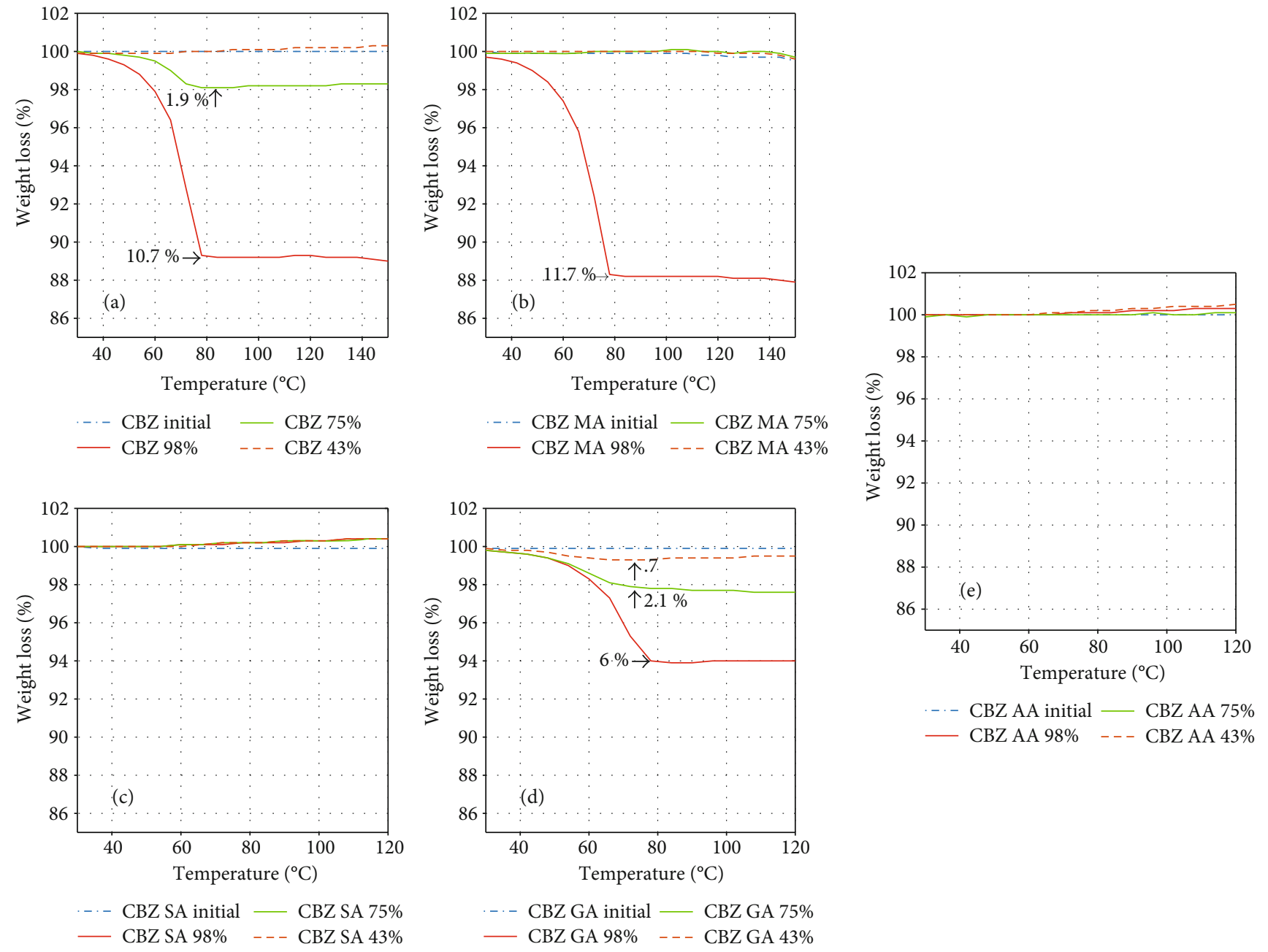

Figure 5: TGA thermograms of (i) pure CBZ, (ii) CBZ-MA, (iii) CBZ-SA, (iv) CBZ-GA, and (v) CBZ-AA.

TABLE 3: The \% weight loss of pure CBZ, CBZ-MA, CBZ-SA, CBZGA, and CBZ-AA.

\begin{tabular}{lcc}
\hline Samples & \% relative humidity & \% weight loss \\
\hline \multirow{2}{*}{ CBZ } & 43 & - \\
& 75 & 1.9 \\
& 98 & 10.7 \\
CBZ-MA & 43 & - \\
& 75 & - \\
& 98 & 11.7 \\
CBZ-SA & 43 & - \\
& 75 & - \\
& 98 & - \\
CBZ-GA & 43 & 0.7 \\
& 75 & 2.1 \\
& 98 & 6 \\
CBZ-AA & 43 & - \\
& 75 & - \\
\hline
\end{tabular}

98\% $\mathrm{RH}$, respectively, while stable at $43 \% \mathrm{RH}$ condition as shown in Figure 5 and Table 3. So, overall, CBZ-RH stability has been improved by cocrystallization. It was observed that CBZ-GA is the most unstable cocrystal with highest solubility and improved dissolution. It was also seen in the previous studies that a highly soluble cocrystal is least stable [34]. A pure CBZ and cocrystals were evaluated for oral bioavailability in rabbits along with marketed product to assess the spacer group effect and to confirm the in vitro improvement of $\mathrm{CBZ}$ cocrystals translation into in vivo pharmacokinetic benefit. The plasma drug concentration vs. time profile and pharmacokinetic parameters are given in Figure 6 and Table 4, respectively. The results of in vivo pharmacokinetic study showed that highest oral bioavailability $(64 \pm 2.2 \mu \mathrm{g} \mathrm{h} / \mathrm{mL})$ and $C_{\max }(7 \pm 0.35 \mu \mathrm{g} / \mathrm{mL})$ were observed by CBZ-GA. The oral bioavailability of CBZ-GA was significantly higher than pure CBZ $(29.87 \pm 2.1 \mu \mathrm{gh} / \mathrm{mL})$ and marketed product $(56.03 \pm 1.2 \mu \mathrm{gh} / \mathrm{mL})$. The $\mathrm{AUC}_{0-t}$ of CBZ-MA $(58 \pm 1.8 \mu \mathrm{g} \mathrm{h} / \mathrm{mL})$ was not considerably higher than marketed product. Moreover, the bioavailability and $C_{\max }$ of prepared cocrystals were much higher than pure CBZ. But, no correlation was observed between oral bioavailability and 


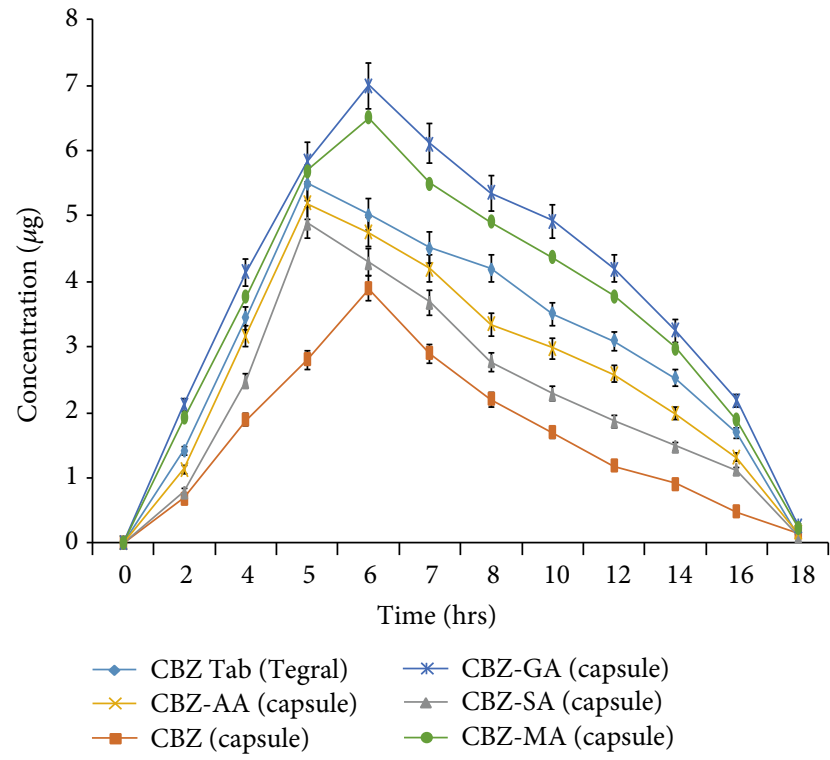

FIGURE 6: In vivo drug release profile of pure CBZ, cocrystals, and marketed product.

TABLE 4: Pharmacokinetic parameters of pure CBZ, cocrystals, and marketed product.

\begin{tabular}{lccc}
\hline Formulation & $\begin{array}{c}\mathrm{AUC}_{0-18} \\
(\mu \mathrm{gh} / \mathrm{mL})\end{array}$ & $\begin{array}{c}C_{\max } \\
(\mu \mathrm{g} / \mathrm{mL})\end{array}$ & $T_{\max }(\mathrm{h})$ \\
\hline CBZ tab (marketed) & $56.03 \pm 1.2$ & $5.5 \pm 0.32$ & $5 \pm 0.72$ \\
CBZ (capsule) & $29.87 \pm 2.1$ & $3.9 \pm 0.27$ & $6 \pm 0.22$ \\
CBZ-SA (capsule) & $47.81 \pm 2.00$ & $4.9 \pm 0.31$ & $5 \pm 0.23$ \\
CBZ-AA (capsule) & $50.17 \pm 1.00$ & $5.2 \pm 0.21$ & $5 \pm 0.21$ \\
CBZ-GA (capsule) & $64 \pm 2.2$ & $7 \pm 0.35$ & $6 \pm 0.27$ \\
CBZ-MA (capsule) & $58 \pm 1.8$ & $6.5 \pm 0.30$ & $6 \pm 0.38$ \\
\hline
\end{tabular}

spacer group of coformers. However, the in vivo study results exhibit that solubility and dissolution-restricted oral bioavailability can be enhanced by the cocrystallization technique.

\section{Conclusion}

The present research work concludes the influence of spacer group (varying aliphatic chain length) on solubility, dissolution, RH stability, and oral bioavailability of CBZ cocrystals with dicarboxylic acids coformers. According to the reported results, a good improvement in dissolution is shown by CBZGA being the most soluble and unstable cocrystal. The low soluble cocrystal is CBZ-SA which does not show improved dissolution. Hence, the order of enhanced solubility and dissolution is CBZ-GA $(n=3)>$ CBZ-MA $(n=1)>$ CBZ-AA $(n=4)>$ pure CBZ > CBZ-SA $(n=2)$. The highly and poorly soluble cocrystals show the highest and lowest dissolution, respectively. So, solubility and dissolution are consistent with each other. Similarly, the increased order of oral bioavailability observed is CBZ-GA $(n=3)>$ CBZ-MA $(n=4)>$ mar-
TABLE 5: FT-IR peaks summary of individual components and respective cocrystals.

\begin{tabular}{lccc}
\hline Compounds & Peaks $\left(\mathrm{cm}^{-1}\right)$ & Groups & Inference \\
\hline CBZ & 3465 & N-H stretching & - \\
& 1675 & C=O stretching & - \\
AA & 1688 & C=O stretching & - \\
GA & 1686 & C=O stretching & - \\
SA & 1682 & C=O stretching & - \\
MA & 1696 & C=O stretching & - \\
& 3355 & N-H stretching & Cocrystal formed \\
CBZ-AA & 1698 & C=O stretching & \\
& 3482 & N-H stretching & Cocrystal formed \\
CBZ-GA & 1712 & C=O stretching & \\
& 3469 & N-H stretching & Cocrystal formed \\
CBZ-SA & 1698 & C=O stretching & \\
& 3481 & N-H stretching & Cocrystal formed \\
CBZ-MA & 1701 & C=O stretching & \\
& &
\end{tabular}

Abbreviations: CBZ: carbamazepine, MA: malonic acid, SA: succinic acid, GA: glutaric acid, AA: adipic acid.

keted product $>$ CBZ-AA $(n=2)>$ CBZ-SA $(n=1)>$ pure CBZ. In conclusion, no consistency of spacer group effects is seen on physicochemical properties (solubility and dissolution) and oral bioavailability of CBZ cocrystals. In other words, increasing hydrophobicity of coformer has no regular effect on cocrystals physicochemical properties and oral bioavailability.

\section{Data Availability}

Data used to support this study finding have been included in the article and could be provided upon request from first author Muhammad Wasim (wassypharmacist@gmail.com).

\section{Conflicts of Interest}

Authors declare that there is no conflict of interest.

\section{Acknowledgments}

The authors would like to acknowledge the Higher Education Commission (HEC), Pakistan, for supporting the six-month research visit to Université Catholique de Louvain, Belgium, under the International Research Support Initiative Program. The authors would also like to gratefully acknowledge the invaluable services and assistance provided by Centralized Resources Laboratory (CRL), Peshawar University for in vivo pharmacokinetic study. Moreover, authors highly acknowledge Dr. Muhammad Hassham Hassan Bin Asad (KFU, Russia; CUI, Pakistan) for his valuable support to publish this research work.

\section{Supplementary Materials}

Figure S1: it contains XRPD patterns of different forms of CBZ and CBZ-MA. (Supplementary Materials) 


\section{References}

[1] D. D. Gadade and S. S. Pekamwar, "Pharmaceutical cocrystals: regulatory and strategic aspects, design and development," Advanced pharmaceutical bulletin, vol. 6, no. 4, pp. 479-494, 2016.

[2] D. Douroumis, S. A. Ross, and A. Nokhodchi, "Advanced methodologies for cocrystal synthesis," Advanced Drug Delivery Reviews, vol. 117, pp. 178-195, 2017.

[3] M. Chahkandi, M. H. Bhatti, U. Yunus et al., "Novel cocrystal of $N$-phthaloyl- $\beta$-alanine with 2,2-bipyridyl: Synthesis, computational and free radical scavenging activity studies," Journal of Molecular Structure, vol. 1152, pp. 1-10, 2018.

[4] S. F. Silva Filho, A. C. Pereira, J. M. G. Sarraguça et al., "Synthesis of a glibenclamide cocrystal: full spectroscopic and thermal characterization," Journal of Pharmaceutical Sciences, vol. 107, no. 6, pp. 1597-1604, 2018.

[5] X. Wang, S. Du, R. Zhang, X. Jia, T. Yang, and X. Zhang, "Drug-drug cocrystals: opportunities and challenges," Asian Journal of Pharmaceutical Sciences, vol. 2, 2020.

[6] S. S. A. Abidi, Y. Azim, S. N. Khan, and A. U. Khan, "Sulfaguanidine cocrystals: synthesis, structural characterization and their antibacterial and hemolytic analysis," Journal of Pharmaceutical and Biomedical Analysis, vol. 149, pp. 351-357, 2018.

[7] F. Cao, G. L. Amidon, N. Rodríguez-Hornedo, and G. E. Amidon, "Mechanistic basis of cocrystal dissolution advantage," Journal of Pharmaceutical Sciences, vol. 107, no. 1, pp. 380389, 2018.

[8] G. Kuminek, K. L. Cavanagh, M. F. M. da Piedade, and N. Rodríguez-Hornedo, "Posaconazole cocrystal with superior solubility and dissolution behavior," Crystal Growth \& Design, vol. 19, no. 11, pp. 6592-6602, 2019.

[9] A. Shayanfar, K. Asadpour-Zeynali, and A. Jouyban, "Solubility and disso4lution rate of a carbamazepine-cinnamic acid cocrystal," Journal of Molecular Liquids, vol. 187, pp. 171-176, 2013.

[10] J.-M. Li, X.-L. Dai, G.-J. Li, T.-B. Lu, and J.-M. Chen, "Constructing anti-glioma drug combination with optimized properties through cocrystallization," Crystal Growth \& Design, vol. 18, no. 8, pp. 4270-4274, 2018.

[11] M. Ullah, I. Hussain, and C. C. Sun, "The development of carbamazepine-succinic acid cocrystal tablet formulations with improvedin vitroandin vivoperformance," Drug Development and Industrial Pharmacy, vol. 42, no. 6, pp. 969-976, 2016.

[12] N. Rodríguez-Hornedo, S. J. Nehm, K. F. Seefeldt, Y. PagánTorres, and C. J. Falkiewicz, "Reaction crystallization of pharmaceutical molecular complexes," Molecular Pharmaceutics, vol. 3, no. 3, pp. 362-367, 2006.

[13] R. Thakuria, A. Delori, W. Jones, M. P. Lipert, L. Roy, and N. Rodríguez-Hornedo, "Pharmaceutical cocrystals and poorly soluble drugs," International Journal of Pharmaceutics, vol. 453, no. 1, pp. 101-125, 2013.

[14] H. Zhang, Y. Zhu, N. Qiao, Y. Chen, and L. Gao, "Preparation and characterization of carbamazepine cocrystal in polymer solution," Pharmaceutics, vol. 9, no. 4, 2017.

[15] E. R. Gaikwad, S. S. Khabade, T. B. Sutar, M. R. Bhat, and S. A. Payghan, "Three-dimensional Hansen solubility parameters as predictors of miscibility in cocrystal formation," Asian Journal of Pharmaceutics, vol. 11, no. 4, pp. 302-318, 2017.

[16] A. Shevchenko, I. Miroshnyk, L.-O. Pietilä et al., "Diversity in itraconazole cocrystals with aliphatic dicarboxylic acids of varying chain length," Crystal Growth \& Design, vol. 13, no. 11, pp. 4877-4884, 2013.

[17] Y.-H. Luo and B.-W. Sun, "Pharmaceutical co-crystals of pyrazinecarboxamide (PZA) with various carboxylic acids: crystallography, hirshfeld surfaces, and dissolution study," Crystal Growth \& Design, vol. 13, no. 5, pp. 2098-2106, 2013.

[18] K. Moribe, A. Nagai, Y. Hagiwara, W. Limwikrant, K. Higashi, and K. Yamamoto, "Carbamazepine-dicarboxylic acid cocrystal formation induced by multicomponent cogrinding and exchange reaction of dicarboxylic acids," Journal of the Society of Powder Technology, Japan, vol. 49, no. 3, pp. 184-190, 2012.

[19] S. L. Childs, P. A. Wood, N.. Rodríguez-Hornedo, L. S. Reddy, and K. I. Hardcastle, "Analysis of 50 crystal structures containing carbamazepine using the materials module of mercury CSD," Crystal Growth \& Design, vol. 9, no. 4, pp. 1869-1888, 2009.

[20] G. Dutkiewicz, E. Dutkiewicz, and M. Kubicki, "Even-odd effect in the co-crystals of pyrazine and dicarboxylic acids," Structural Chemistry, vol. 26, no. 1, pp. 247-259, 2015.

[21] B. R. Jali and J. B. Baruah, "Cocrystals of 2,4-diamino-6-phenyl-1,3,5-triazine with dicarboxylic acids," Journal of Chemical Crystallography, vol. 43, no. 10, pp. 531-537, 2013.

[22] A. C. Moffat, M. D. Osselton, and B. Widdop, Clarke's analysis of drugs and poisons, vol. 3, no. 4, 2011Pharmaceutical press London, 2011.

[23] M. B. Hickey, M. L. Peterson, L. A. Scoppettuolo et al., "Performance comparison of a co-crystal of carbamazepine with marketed product," European Journal of Pharmaceutics and Biopharmaceutics, vol. 67, no. 1, pp. 112-119, 2007.

[24] S. L. Childs, N. Rodríguez-Hornedo, L. S. Reddy et al., "Screening strategies based on solubility and solution composition generate pharmaceutically acceptable cocrystals of carbamazepine," CrystEngComm, vol. 10, no. 7, pp. 856-864, 2008.

[25] W. Limwikrant, A. Nagai, Y. Hagiwara, K. Higashi, K. Yamamoto, and K. Moribe, "Formation mechanism of a new carbamazepine/malonic acid cocrystal polymorph," International Journal of Pharmaceutics, vol. 431, no. 1-2, pp. 237240, 2012.

[26] Z. Rahman, R. Samy, V. A. Sayeed, and M. A. Khan, "Physicochemical and mechanical properties of carbamazepine cocrystals with saccharin," Pharmaceutical Development and Technology, vol. 17, no. 4, pp. 457-465, 2012.

[27] N. A. Ramle, S. A. Rahim, N. Anuar, and O. EI-Hadad, "Solubility of carbamazepine co-crystals in ethanolic solution," in Conference Proceedings, vol. 1879no. 1AIP Publishing LLC.

[28] D. D. Bavishi and C. H. Borkhataria, "Spring and parachute: how cocrystals enhance solubility," Progress in Crystal Growth and Characterization of Materials, vol. 62, no. 3, pp. 1-8, 2016.

[29] H. Zhang, Q. Yin, Z. Liu et al., "An odd-even effect on solubility of dicarboxylic acids in organic solvents," The Journal of Chemical Thermodynamics, vol. 77, pp. 91-97, 2014.

[30] H. Yamashita and C. C. Sun, "Improving dissolution rate of carbamazepine-glutaric acid cocrystal through solubilization by excess coformer," Pharmaceutical Research, vol. 35, no. 1, 2017.

[31] J. Cadden, W. T. Klooster, S. J. Coles, and S. Aitipamula, "Cocrystals of leflunomide: design, structural, and physicochemical evaluation," Crystal Growth \& Design, vol. 19, no. 7, pp. 3923-3933, 2019.

[32] Y. Huang, G. Kuminek, L. Roy, K. L. Cavanagh, Q. Yin, and N. Rodríguez-Hornedo, "Cocrystal solubility advantage 
diagrams as a means to control dissolution, supersaturation, and precipitation," Molecular Pharmaceutics, vol. 16, no. 9, pp. 3887-3895, 2019.

[33] Y. Gao, H. Zu, and J. Zhang, "Enhanced dissolution and stability of adefovir dipivoxil by cocrystal formation," Journal of Pharmacy and Pharmacology, vol. 63, no. 4, pp. 483-490, 2011.

[34] D. J. Good and N.. Rodríguez-Hornedo, "Solubility advantage of pharmaceutical cocrystals," Crystal Growth \& Design, vol. 9, no. 5, pp. 2252-2264, 2009. 